Educación y TIC

Educação e TIC

Education and ICT

\title{
LAS REDES SOCIALES: UNA ESTRATEGIA PEDAGÓGICA PARA INCENTIVAR EL EMPRENDIMIENTO
}

REDES SOCIAIS: UMA ESTRATÉGIA PEDAGÓGICA PARA ESTIMULAR O EMPREENDEDORISMO²

SOCIAL NETWORKS: A PEDAGOGICAL STRATEGY TO ENCOURAGE ENTREPRENEURSHIP ${ }^{3}$

Alicia Morales ${ }^{4}$ y Héctor Alirio Corredor ${ }^{5}$

Universidad Cooperativa de Colombia

\section{CIENCIA Y PODER AÉREO}

ISSN 1909-7050 / E- ISSN 2389-2468 / Volumen 11/ Enero-diciembre de 2016/ Colombia/ Pp. 242-255 Recibido: 23/09/2015

Aprobado evaluador interno: 10/11/2015

Aprobado evaluador externo: 21/01/2016

Doi: http://dx.doi.org/10.18667/cienciaypoderaereo.522 
Para citar este artículo:

Morales, A., \& Corredor, H.. (2016). Las redes sociales: una estrategia pedagógica para incentivar el emprendimiento. Ciencia y Poder Aéreo, 11 (1), 242-255. Doi: http:// dx.doi.org/10.18667/cienciaypoderaereo.522

\begin{abstract}
' Artículo de reflexión, derivado del proyecto de investigación: desarrollo de una estrategia pedagógica basada en redes sociales para incentivar el emprendimiento de los estudiantes de décimo y undécimo de la modalidad asistencia administrativa del Colegio Fernando Mazuera Villegas, ubicado en Bogotá D.C., localidad de Bosa. Maestría en Educación 2016. Universidad Cooperativa de Colombia.
\end{abstract}

${ }^{2}$ Artigo de reflexão, derivado do projeto de pesquisa: Desenvolvimento de uma Estratégia Pedagógica baseada Das Redes Sociais para Incentivar o Empreendedorismo dos estudantes de decimo e decimo primeiro graus, da modalidade de Assistência Administrativa do Colégio Fernando Mazuera Villegas localizado em Bogotá D.C, Bosa. Mestrado em Educação 2016. Universidade Cooperativa de Colômbia.

${ }^{3}$ Reflection article, derived from the research project: Development of a Pedagogical Strategy Based On Social Networks to Promote Entrepreneurship of Students of the Tenth and Eleventh Grade of the assistance administrative modality of the Fernando Mazuera Villegas School located in Bogota D.C, Bosa. Masters in Education 2016. Cooperative University of Colombia.

${ }^{4}$ Magíster en Educación. Especialista en Gerencia de Proyectos Educativos. Administradora Financiera, Docente Educación Media Especializada, Colegio Fernando Mazuera Villegas. Bogotá, Colombia. Correo electrónico: aliciamoraro@gmail.com

${ }^{5}$ Magíster en Educación. Especialista en Gerencia de Proyectos Educativos. Geógrafo. Docente Ciencias Sociales de la Institución Educativa CEDID, Ciudad Bolívar. Bogotá, Colombia. Correo electrónico: hectorcorredor@yahooo.com.ar

${ }^{6}$ Haciendo referencia con éste término a las experiencias que se viven fuera del aula en el mundo laboral.
Resumen: tras la preocupación por el uso que los jóvenes del Colegio Fernando Mazuera Villegas, (Bogotá D.C., Colombia), dan a las redes sociales y el desconocimiento de todas las herramientas de provecho que éstas ofrecen, el grupo investigador busca desarrollar una estrategia, que permita canalizar la atención de los estudiantes, en programas que forjen verdaderas actitudes y aptitudes de emprendimiento alrededor del uso asertivo de las mismas. El presente artículo describe las diferentes etapas por las que la investigación atraviesa para gestionar un programa de calidad que brinde mecanismos para impulsar y proyectar con innovación, ideas emprendedoras a través del uso adecuado de las redes sociales, en el aula de clase. Su desarrollo se enmarca dentro del paradigma cualitativo, dado que en este tipo de investigaciones, la realidad es la persona y el entorno que le rodea de forma directa. El enfoque metodológico utilizado, recorre las etapas de descripción e interpretación para finalmente proponer una estrategia pedagógica, que permita desmantelar el verdadero potencial de las redes sociales, aprovechando el interés innato que las mismas despiertan en la juventud Colombiana. Los resultados, demostraron impacto positivo en el cambio de hábitos para el uso de las redes sociales, descubriendo, como ambientes virtuales que manipulan diariamente, esconden grandes beneficios para su propio proyecto de vida y relacionando exitosamente los conocimientos que adquieren en el aula de clase con las necesidades propias del "mundo real"

Palabras clave: emprendimiento; estrategia pedagógica; herramientas virtuales; ideas de negocio; proyecto de vida; redes sociales.

Resumo: após da preocupação pelo uso que os jovens do Colégio Fernando Mazuera Villegas, (Bogotá D.C., Colômbia), fazem das redes sociais e o desconhecimento de todas as ferramentas para aproveitar que elas oferecem, a turma de pesquisa, procura desenvolver uma estratégia que permita focar a atenção dos estudantes em programas que forneçam atitudes verdadeiras y aptidões de empreendedorismo ao redor do uso assertivo das mesmas. Esse artigo descreve as diferentes etapas pelas que passa a pesquisa para administrar um programa de qualidade que dê mecanismos para impulsionar e projetar com inovação, ideias empreendedoras através do uso correto das redes sociais no salão de aulas. Seu desenvolvimento fica dentro do paradigma qualitativo, já que neste tipo de pesquisas, a realidade é a pessoa e o ambiente que a rodeia de maneira direta. O foco metodológico utilizado percorre as etapas de descrição e interpretação para, finalmente, propor uma estratégia pedagógica que vai deixar desmantelar o verdadeiro potencial das redes sociais aproveitando o interesse inato que elas causam na juventude colombiana. Os resultados, mostraram impacto positivo na mudança dos hábitos para o uso das redes sociais descobrindo, como ambientes virtuais que manipulam no dia a dia e escondem grandes benefícios para seu próprio projeto de vida e relacionado com grande sucesso os conhecimentos que ganham no salão de aulas com necessidades próprias do "mundo real".

Palavras-chave: empreendedorismo; estratégia pedagógica; ferramentas virtuais; ideias de negócio; projeto de vida; redes sociais.

Abstract: Because of the concern about the use that the young people of the Fernando Mazuera Villegas School, (Bogota D.C., Colombia), give to social networks and the ignorance of all the tools of profit these offer, the research group seeks to develop a strategy that will channel the attention of students in programs that forge true attitudes and entrepreneurship skills around the assertive use of them. This article describes the different stages through which the research goes through to manage a quality program that provides mechanisms to promote and project with innovation, entrepreneurial ideas through the proper use of social networks in the classroom. Its development is framed within the qualitative paradigm, given that in this type of research, the reality is the person and the environment that surrounds him directly. The methodological approach used traverses the stages of description and interpretation to finally propose a pedagogical strategy that allows dismantling the true potential of social networks, taking advantage of the innate interest that these awaken in the Colombian youth. The results showed a positive impact on the change of habits for the use of social networks, finding how virtual environments that they use daily, hide great benefits for their own life project and successfully relate the knowledge they acquire in the classroom with the needs of the "real world".

Key Words: Entrepreneurship; Pedagogic Strategy; Virtual Tools; Business Ideas; Life Project; Social Networks. 


\section{Introducción}

La humanidad, ha venido evidenciado hechos que cambian el rumbo de la historia. Hoy somos agentes activos de un proceso de transformación determinado por avances tecnológicos, que ofrecen un sin número de opciones para la ejecución de las tareas exigidas en cada uno de los roles, desempeñados dentro de una sociedad. Sociedad en la que se debe evidenciar la integralidad de: "un individuo, una empresa, una sociedad y un ambiente" (Waldemar, 2002).

Facebook, Google+, YouTube, Twitter, Linkedln, Tumblr, Instagram, WhatsApp, Google Adsense, han sido la revolución en la forma como emerge esa integralidad. Todas, surgen alrededor de la necesidad innata que el hombre tiene por comunicarse con sus semejantes. "El hombre necesita comunicarse con los demás para intercambiar de todo: comida, utensilios, trabajo, amistad, afección, sentimientos, opiniones" (Baiget, 2009), situación que hasta ahora estaba limitada, y con la aparición de las redes sociales el entramado social se transforma.

El Ministerio de las TIC $^{7}$ y la consultora Ipsos Napoleón Franco presentaron el estudio de Consumo Digital de los colombianos el 14 de febrero de 2013. Los resultados de dicho estudio demostraron que la mayoría (80\%) usa internet. De éste porcentaje, el 54\% lo hace todos los días y pasan un promedio aproximado de 3 horas navegando. Además, que los estratos 1 y 2 presentaron un aumento del 17\% en el uso de la red comparado con el año 2010.

8 de cada 10 colombianos utilizan redes sociales. El 70 $\%$ tiene una cuenta activa, siendo Facebook la más usada con un 98\%, resultados tendientes al crecimiento, pues Twitter e Instagram empiezan a posicionarse. En conclusión, el estudio evidencia el incremento constante del acceso a internet y el interés que el mismo ha despertado en la población colombiana, la encuesta señala crecimiento en el acceso a redes sociales (del 73\% al 81\%) y búsqueda de información a través de diversos buscadores (del 68\% al 74\% en 2013).

Los anteriores resultados son muy alentadores, sí se tratara de analizar el acceso que los colombianos tienen al internet y redes sociales. Además, de ser una muestra de los esfuerzos gubernamentales, porque la población colombiana tenga acceso a las nuevas tecnologías de la información; sin embargo, la preocupación actual, no es el acceso a ellas, sino la ausencia de hábitos sanos en la utilización de las mismas. El uso inadecuado de la información, y la carencia de conocimiento sobre el beneficio que

\footnotetext{
7 Tecnologías de la Información y la Comunicación
}

ellas ofrecen, afectan drásticamente las relaciones interpersonales, distorsionando la cadena armónica que se debe generar, para poder alcanzar el desarrollo sistémico de una sociedad que propenda por su bienestar y el del ambiente.

Son muchas las estrategias que se han implementado para que la población tenga acceso a éstas tecnologías, pero son muy pocas las estrategias "en el aula de clase", para desmantelar el verdadero potencial que éstas ofrecen en la construcción de conocimiento, y como centro de la investigación, para mejorar la calidad de vida del joven estudiante a través del uso de las herramientas, que permiten promover bienes y servicios en éste contexto sin límites geográficos y culturales.

Un análisis exhaustivo sobre la documentación existente alrededor del tema, permitieron reunir material muy valioso de investigaciones realizadas a categorías asociadas a la investigación:

Herrera (2009), cuyo cuestionamiento gira en torno a establecer cuáles son los aportes de la literatura más reciente acerca de los efectos de las redes sobre el emprendimiento, realiza descripciones teóricas muy enriquecedoras acerca de cómo el contenido y la estructura de las redes sociales contribuyen al éxito de la actividad del emprendedor.

Arias y Castillo (2011), reúnen elementos sobre nuevas visiones de la relación entre educación, uso de las TIC y emprendimiento, como forma de abordar el mundo global al que el estudiante se enfrenta, en su investigación: la educación para el emprendimiento y empresarismo virtual.

Núñez (2012) plantea: ¿cómo aplicar de manera práctica en las aulas el desarrollo, manejo y aplicación de las redes sociales? y señala en su investigación, la profesión en el aula. investigar y mejorar el trabajo del futuro, cómo las empresas utilizan cada vez más variadas herramientas digitales para comunicarse con sus públicos en comunicación bidireccional y Enciso y Sánchez M. (2012), quienes establecen como pregunta de investigación ¿cómo gestionar las tecnologías de la información de manera que se aprovechen al máximo los recursos, en pro de alcanzar los objetivos empresariales? Proporcionan información relevante sobre cómo el emprendimiento puede surgir desde estas herramientas, y cómo, el espíritu emprendedor encuentra en el uso de las redes sociales, amplio campo de acción y proyección en la construcción de proyectos de vida productivos.

Investigaciones que permitieron evidenciar correlación entre tres aspectos: educación, comunicación y producción. Confirmando la necesidad educativa de plantear 
una estrategia pedagógica que engrane asertiva y funcionalmente la "comunicación", por la forma cómo las redes sociales revolucionan el entramado social y "producción" por la necesidad educativa de "articular la educación con el mundo laboral" (Mineducación, 2006).

Con el paso del tiempo, se podrán reducir las diferencias sociales, si los futuros usuarios de la web $4.0^{8}$ además de poder acceder al internet, adquieren conocimientos que les permitan desarrollar actitudes y aptitudes para seleccionar, cribar e identificar el verdadero valor de construir y compartir conocimientos que contribuyan a su formación profesional y por ende a la ampliación de oportunidades en sus venideros años laborales.

Razón por la que el objetivo de la investigación, es desarrollar una estrategia pedagógica que incentive la cultura emprendedora, aprovechando el alcance que las herramientas de las redes sociales ofrecen para tal fin.

De acuerdo a Llera (2003), "para que las TIC desarrollen todo su potencial de transformación (...) deben integrarse en el aula y convertirse en un instrumento cognitivo capaz de mejorar la inteligencia y potenciar la aventura de aprender". En consecuencia, dicha condición, indudablemente debe ser impulsada desde la escuela, para que las posibilidades del joven no se limiten a las relaciones sociales en el aula de clase sino que trascienda fronteras lingüísticas, culturales y económicas.

Si bien es cierto, que con el paso del tiempo, la Escuela se ha esforzado por forjar individuos integrales que tengan acceso a las nuevas tecnologías, también es cierto que debido a la velocidad con la que surgen los últimos avances tecnológicos, se está quedando corta en brindar conocimientos que le permitan al joven estudiante desenvolverse con asertividad en la utilización de los medios digitales como herramienta para gestionar su proyecto de vida.

A partir de lo anteriormente planteado surge la pregunta de investigación:

¿Cómo utilizar en las aulas de clase, las redes sociales para desarrollar competencias de emprendimiento que contribuyan a la gestión del proyecto de vida?

"La pedagogía no debe amoldarse sin más a la tecnología, pero tampoco debe dejar de aprovechar las posibilidades innovadoras que ésta le ofrece" (Graells, 2005). La

\footnotetext{
${ }^{8}$ Web 4.0, o web ubicua donde el objetivo primordial será unir las inteligencias donde tanto las personas como las cosas se comuniquen entre sí para generar la toma de decisiones (Castilla, 2011)
}

web se puede utilizar como medio para incursionar en el mundo productivo, potenciar habilidades individuales a través de la participación en comunidades colaborativas y como plataforma publicitaria de ideas innovadoras en otras tantas ventajas. Es todo un entorno sistémico que facilita la generación de oportunidades para crecer individual y colectivamente. Desafortunadamente, el conocimiento sobre ésta tendencia no está en el aula de clase sino que es enrutada sólo a segmentos exclusivos de la población.

Aparece un elemento de división social mucho más importante que la conectividad técnica, y es la capacidad educativa y cultural de utilizar Internet. Una vez que toda la información está en la red, una vez que el conocimiento está en la red, el conocimiento codificado, pero no el conocimiento que se necesita para lo que se quiere hacer, de lo que se trata es de saber dónde está la información, cómo buscarla, cómo procesarla, cómo transformarla en conocimiento específico para lo que se quiere hacer. Esa capacidad de aprender a aprender, esa capacidad de saber qué hacer con lo que se aprende, esa capacidad es socialmente desigual y está ligada al origen social, al origen familiar, al nivel cultural, al nivel de educación. Es ahí donde está, empíricamente hablando, la divisoria digital en estos momentos (Jóvenes en el siglo XXI).

Es papel de la Escuela, intervenir en este proceso. Por ello, la investigación concluye con la inclusión, en los ambientes educativos, de experiencias que generan en los jóvenes estudiantes culturas digitales, y no solamente en cuánto a hábitos sanos se refiere, también a la explotación ${ }^{9}$ de los instrumentos que la web 2.0 pone a disposición del usuario para proyectar de manera panorámica su visión profesional.

En el G-20 se calcula que la oportunidad de negocio en la red asciende a los 4,2 billones de dólares (Portafolio, 2012). En éste artículo de El Portafolio, se revelan estudios que demuestran la manera como está creciendo la industria en internet. Anualmente, en los países desarrollados se constituyen 150 mil empresas cuyo objeto económico gira alrededor de actividades relacionadas con el internet, y al contrario de lo que se creía, el internet está contribuyendo a nuevas formas para crear empresa y promulgar la venta de bienes y servicios, situación que beneficia las ideas emprendedoras generadas por la juventud colombiana.

La creciente importancia que la web está tomando en la generación de alianzas estratégicas para el desarrollo de

\footnotetext{
${ }^{9}$ En buen uso de la palabra.
} 
la industria, permiten que se derrumbe el imaginario de que la internet contribuye al crecimiento del desempleo en un país, por el contario su influencia va más allá de facilitar la comunicación entre diferentes culturas y sociedades, las 2 mil millones de personas que se conectan (cifra tendiente al crecimiento según estudios realizados por Mckinzey \& Co.) crean una nueva dinámica alrededor de la forma como se hacen negocios y se potencian ideas de emprendimiento situación que tiene un impacto directo en el crecimiento económico de un país.

No se requiere gran capital, está al acceso de todos y todas, y como si fuera poco, los jóvenes pertenecen a la Generación de los bien Ilamados "Nativos Digitales" (Prensky, 2011), lo que les da habilidades innatas para el manejo de nuevas tecnologías que facilitan la ruta hacia la innovación y se convierten en un medio para potenciar el emprendimiento, cualidad que representa la columna vertebral a la hora de desenvolverse en un mundo lleno de nuevos desafíos.

Para Ahumada (2013), el emprendimiento y el autoempleo constituyen algunos de los espacios donde la generación de ocupación laboral se representa como opción alternativa. Es necesario por tanto, diseñar recursos y estrategias que reconduzcan los procesos de empleado a empleador.

Dichas estrategias deben liberar cualidades de autonomía en el trabajador como gestor de su propio itinerario profesional y laboral. Este planteamiento de trabajo por cuenta propia exige manejo de conocimientos diferentes al empleo por cuenta ajena.

La recolección y divulgación pedagógica de dichos conocimientos, se convierten en el fin de la investigación. Razón por la que se elabora un manual cuya estructura está diseñada bajo la interrelación entre las categorías identificadas al comienzo de la investigación: Educación, Comunicación y producción (Waldemar, 2002) y pone a disposición del estudiante conocimientos alrededor de las herramientas que brindan las redes sociales para poner en marcha ideas emprendedoras: diseño de fanpage, WhatsApp para empresas, carrito online, botón PSE, Youtubers, Instagram en la empresa, Twitter for Bussines, Google Adsensey Paypal.

Esta estrategia permite, después de la implementación de instrumentos de recolección de información, medir cualitativamente y cuantitativamente el impacto que causa el descubrir los beneficios que para su propio proyecto de vida tienen las redes sociales desarrollando técnicas que promueven su actitud emprendedora y como resultado, se apropian de habilidades y destrezas que en su futuro le permitirán generar beneficios económicos. Aspecto que contribuye al mejoramiento y estilo de su calidad de vida, convirtiéndolo en un agente activo de transformación dentro de una dinámica en constante crecimiento científico, espiritual y productivo.

\section{Marco teórico}

El marco teórico se convierte en el respaldo argumentativo de cada una de las situaciones planteadas en el proyecto de investigación, razón por la que se consultaron los expertos en las categorías asociadas a la problemática, reuniendo sus apreciaciones alrededor del contexto educativo, las redes sociales, el potencial productivo que en ellas se alberga y el uso pedagógico de las mismas.

La triangulación se realiza entonces, sincronizando i) comunicación, ii) producción y iii) educación, respectivamente: categorías seleccionadas desde los 14 subsistemas planteados por Waldemar de Gregory en su libro Capital Intelectual (2002). Herramienta que permitió establecer los debidos enlaces, para poder encontrar la relación entre ellos, obteniendo como resultado una matriz (ver Tabla 1) que vislumbra la manera de utilizar pedagógicamente las redes sociales para potenciar en los jóvenes cultura emprendedora y como beneficio, proporcionar herramientas que les permita incursionar asertivamente en el mundo laboral.

\section{Educación}

\section{Media técnica fortalecida}

Alrededor del proyecto media técnica fortalecida, gira toda una comunidad educativa cuyos esfuerzos están orientados hacia la formación de estudiantes con competencias que les permita desenvolverse con asertividad en el mundo productivo. Para tal fin, El Ministerio de Educación y la Secretaría Distrital, proponen como objetivo del mismo:

Transformar y fortalecer la educación media distrital mediante la consolidación de una oferta diversa, electiva y homologable con educación superior que promueva la continuidad de los estudiantes en este nivel educativo y genere para ellos mayores oportunidades en el mundo socio productivo (Distrital, 2015).

Además de incluir la formación por competencias en los estudiantes como un elemento básico para mejorar la calidad de la educación; por tanto, es un esfuerzo que debe quedar consignado en el plan de mejoramiento ins- 
Tabla 1

Triangulación Marco Teórico

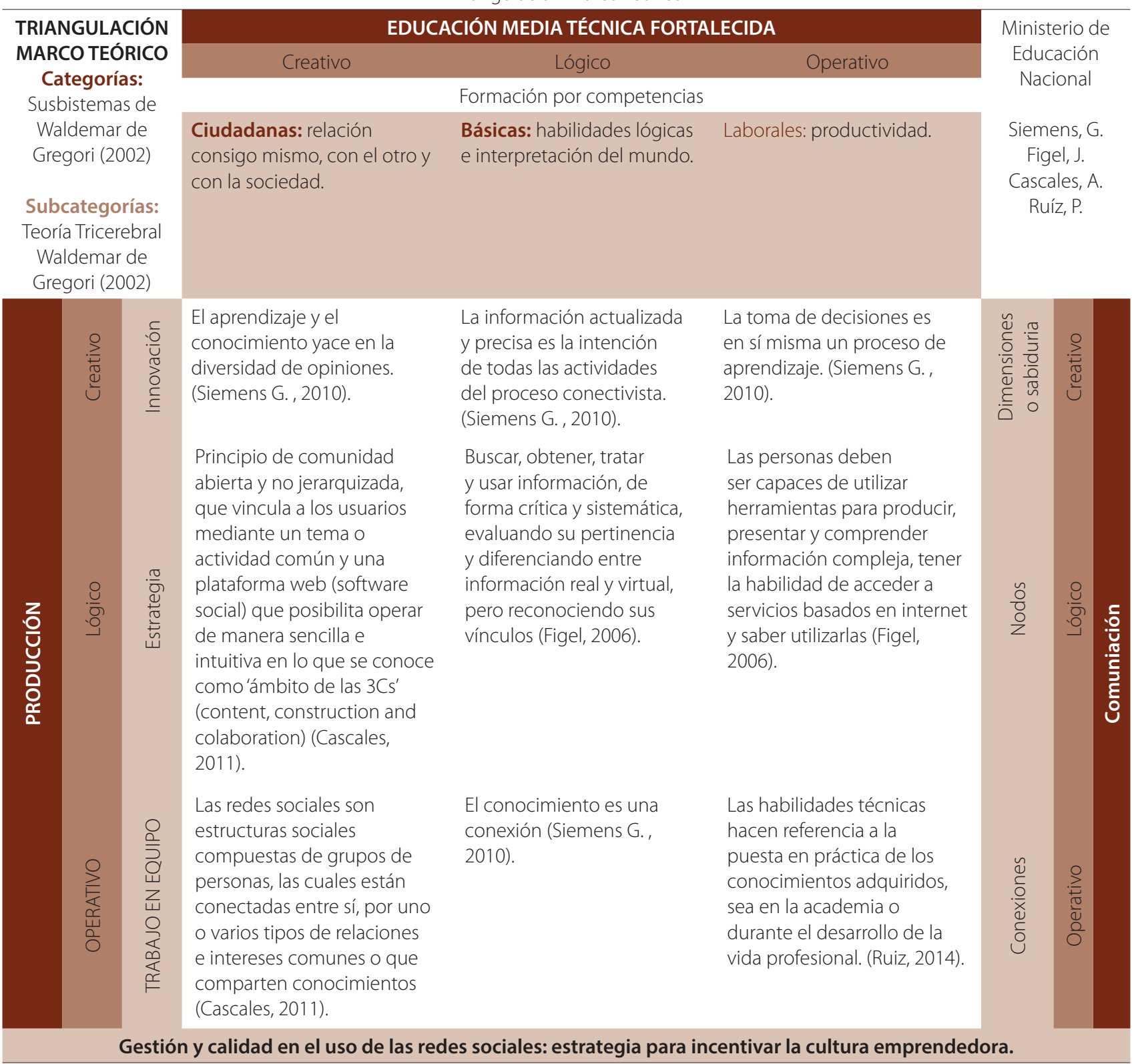

titucional. Se requiere de un enfoque que dé paso a una educación más integradora, que articule la teoría y la práctica, y garantice aprendizajes aplicables a la vida cotidiana (Distrital, 2015).

A continuación se relacionan las razones específicas por las que el Ministerio de Educación considera necesaria la formación por Competencias Laborales (Mineducación, 2006).

Las organizaciones productivas han experimentado sustanciales cambios, originados por la com- petitividad exigida en los mercados globales y en el rápido avance de la tecnología.

Se requieren nuevas organizaciones basadas en redes y equipos de trabajo, que usen tecnologías y procesos flexibles y que tengan en cuenta a los clientes.

El país necesita personas versátiles y polivalentes, que sepan identificar oportunidades para crear negocios, asociarse con otros o generar unidades productivas de carácter asociativo y cooperativo. 
CIENCIA Y PODER AÉREO | Revista Científica de la Escuela de Postgrados de la Fuerza Aérea Colombiana | Vol. 11 | Enero - Diciembre de 2016

Personas que sean capaces de adaptarse a los cambios del entorno, de autodirigirse y autoevaluarse, de relacionarse apropiadamente con otros y de aprender cada vez más sobre su trabajo (Mineducación, 2006).

Los empresarios colombianos han encontrado preocupantes vacíos y limitaciones en los jóvenes egresados de la educación media que aspiran a conseguir un trabajo. Para vincular personal, los empleadores tienen en cuenta, de manera especial, las condiciones personales que involucran competencias clave, como el comportamiento ético y las capacidades para comunicarse, trabajar en equipo y manejar recursos e información.

La formación por competencias laborales no es el desplazamiento de las demás áreas del conocimiento, son complementarios a ese proceso de formación con el propósito de no sólo formar un ser humano con capacidades intelectuales y personales, también con la capacidad productiva que le permita aportar positivamente a su proyecto de vida evidenciándolo a través de su cadena de formación que inicia en la técnica, atraviesa por la tecnológica hasta llegar al nivel profesional.

El país demanda con urgencia personas que tengan la motivación y el entusiasmo necesarios para crear, liderar y sostener empresas propias, para asociarse en cooperativas o para prestar servicio de calidad en una empresa. La actitud emprendedora puede desarrollarse desde los primeros niveles de la escolaridad y conduce a la formación de seres humanos más autónomos, responsables, con habilidades para el manejo de la incertidumbre y el riesgo (Mineducación, 2006).

\section{Producción}

Tal como lo señala Velandia (2006), el subsistema relacionado con la producción integra las operacionales que contribuyen a la extracción, multiplicación, adecuación de materias, servicios o útiles; también está relacionado con la fuerza de trabajo, aptitud y habilidad mental, manual y técnica, creatividad y capacidad de logro. Teniendo en cuenta estos postulados se considera que las temáticas a explorar en este punto son las relacionadas con el emprendimiento y su cultura.

\section{Emprendimiento}

Existen definiciones desde diferentes perspectivas para la palabra emprendedor, en términos generales, se atribuye este apelativo a la persona que hace que las cosas ocurran o sucedan, en este mismo orden de ideas, Rodríguez (2012) señala que desde una mirada política, la perso- na emprendedora es aquella que se esfuerza por convertir sus sueños en realidad, desde una mirada económica es aquel que realiza cambios de recursos de una zona de bajo rendimiento a una de alta productividad, desde una perspectiva operativa consiste en aplicar el talento creador e innovador para engrandecer una empresa ya existente o iniciar la propia, inicia su propio negocio.

La palabra emprendimiento que significa pionero; se refiere a la capacidad de una persona de realizar un esfuerzo adicional para alcanzar una meta; es también utilizada para la persona que inicia una nueva empresa o proyecto. Asimismo, este término se atribuyó a aquellas personas que fueron innovadoras o agregaban valor a un producto ya existente, el emprendimiento es una capacidad de los seres humanos para salir adelante de manera novedosa y con ideas renovadas. Ser emprendedor requiere de habilidades no sólo a nivel individual, sino también colectivo. Una de las razones por las cuales este término se ha vuelto importante en la última década es el resurgimiento de la manera como se han manejado las situaciones económicas y cómo éstas han sido superadas con nuevas ideas (IESE, 2008).

La ley 1014, de enero 26 de 2006, relacionada con el fomento a la cultura del emprendimiento; en sus disposiciones generales señala que el emprendimiento es una manera de pensar y actuar orientada hacia la creación de riqueza. Es una forma de pensar, razonar y actuar centrada en las oportunidades, planteada con visión global y llevada a cabo mediante un liderazgo equilibrado y la gestión de un riesgo calculado, su resultado es la creación de valor que beneficia a la empresa, la economía y la sociedad.

\section{Cultura emprendedora}

La cultura emprendedora puede ser entendida como una serie de procedimientos, requerimientos y formas de actuar que conllevan a la creación y al interés por emprender o comenzar una tarea, motivados ante todo por la voluntad de conseguir unos objetivos claros y precisos.

La cultura emprendedora es aquella que propicia un lugar para trabajar dinámico y creativo (Cameron, 1999), de acuerdo con el autor: una organización con una cultura emprendedora se sostiene por el compromiso hacia la experimentación y la innovación. El énfasis está centrado básicamente en ser el primero y el éxito implica obtener nuevos productos y servicios.

La cultura emprendedora, promueve la incitativa individual, la autonomía y la toma de decisiones de forma participativa. La gente deja sus miedos y se arriesga, los líderes promueven recompensas al éxito y la tolerancia al fracaso 
si se llegase a presentar. Se hace de vital importancia que las personas tengan una actitud verdaderamente positiva en la búsqueda de generar nuevas ideas lo que indiscutiblemente llevara a procesos innovadores. La cultura emprendedora debe ser asumida como una forma o estilo de vida para obtener los mejores resultados.

Características de un buen emprendedor. Se busca que el emprendedor reúna bastantes características favorables para que los logros u objetivos que se proponen en el proyecto o iniciativa lleguen a feliz término, es por tanto, que debe también tener la capacidad de tomar decisiones, trabajar en equipo y ser un buen motivador. En esta línea de ideas, Muñoz (2012) menciona algunos tips o claves para conseguir esas premisas:

- Comunicación: se refiere a brindar la información que permita al equipo entender mejor su trabajo mediante el establecimiento de relaciones respetuosas y constructivas.

- Confianza: Es creer en las capacidades y cualidades de las personas para asumir sus responsabilidades y hacer las cosas bien antes de que lo demuestren.

- Exigencia: la exigencia se genera cuando existe la confianza y seguridad de que las personas harán correctamente sus tareas y así se mantienen expectativas altas sobre los resultados de las mismas.

- Respeto y lealtad: el respeto supone la valoración del otro individuo sin importar su nivel dentro de la jerarquización de una organización, sin críticas destructivas.

- Disponibilidad y asequibilidad: se refiere a promover el contacto con los colaboradores Coherencia: el buen emprendedor debe procurar siempre que sus acciones estén acordes con lo que piensa y dice a sus colaboradores.

- Reconocimiento: promueve en los colaboradores el entusiasmo por hacer mejor su trabajo.

En conclusión es posible señalar que después de analizar los aportes hechos a través del análisis del subsistema producción, la investigación se enriquece y direcciona hacia la consecución de las metas propuestas, los elementos aquí desarrollados muestran que hay mucho por hacer, y se requiere generar estrategias pedagógicas que incentiven una verdadera cultura emprendedora. Uno de los objetivos del proyecto de investigación.

\section{Comunicación}

De acuerdo con Velandia (2006), el subsistema comunicación es la integración de elementos operacionales para la circulación de información u otros bienes, señala también el autor que está relacionado con los medios y estilos de comunicación. Para el desarrollo de la investigación, se tiene en cuenta temática que giran alrededor de: tecnología, competencia digital y redes sociales.

- Tecnología. Tomando como referencia el concepto de tecnología de Ángel Alonso Soto Sarmiento (2000), la tecnología es un saber práctico e interdisciplinario desarrollado a través de la relación teórico - práctica que permite logros de calidad en los procesos aplicados a objetos e instrumentos tecnológicos y a la producción de bienes y servicios con el fin de dar solución a problemas y necesidades humanas.

Siendo así, es importante "dar el siguiente paso y desarrollar un lenguaje y unas herramientas analíticas que le permitan a los grupos sociales adueñarse de su futuro". En palabras de Langdon Winner (2006) citado por Rueda López, "[...] mucho más de lo que hemos reconocido en el pasado, debemos admitir nuestra responsabilidad por lo que estamos haciendo"

El gobierno vasco en su informe Juventud y Tecnologías de la información para las Naciones Unidas (2004) plantea el debate sobre la influencia de las Tecnologías de la Información y la comunicación (TIC) en los jóvenes, haciendo un análisis de los pros y los contras de las TIC.

Al entrar en el debate sobre la influencia de las TIC entre los y las jóvenes encontramos dos posiciones encontradas: una optimista y otra pesimista. Entre los defensores de la primera visión, se reconoce que los jóvenes son la vanguardia en el uso de las TIC. Gracias a las TIC tienen la posibilidad de participar activamente mostrando sus opiniones, críticas y aportaciones en numerosos ámbitos. Tienen acceso a conocimientos, informaciones y experiencias de todo el mundo y al mismo tiempo más información y poder de participación en lo más cercano a ellos. Lo global y lo local se funden e interactúan, hablándose de un nuevo término que las TIC han generado: el ámbito "global".

En investigación desarrollada por Sergio Balardini, con la colaboración de Mariela Mociulsky \& Ximena Díaz Alarcón de Trendsity (2009) y titulado Chic@s y Tecnología. Usos y costumbres de niñas, niños y adolescentes en relación a las TIC (Balardini, 2009) se presentan elementos referentes a los usos y costumbres de los niños y adoles- 
centes con las TIC, de este trabajo se rescatan como fuente fundamental de análisis, algunas recomendaciones que se hacen para todos los actores involucrados en el trabajo de los menores con las herramientas tecnológicas.

De acuerdo con Soto (2000), en el ámbito de lo expresamente educativo, se indica el reto

debe afrontar la escuela, y dentro de ella, el profesor de tecnología adquiere una doble dirección: de un lado, posibilitar desde la educación básica, seres capaces de responder en el futuro al reto de desarrollo tecnológico; y del otro, formar seres conscientes de la necesidad de dar soluciones concretas a problemas reales.

- Competencia digital. Dentro de las capacidades que se deben desarrollar, Figel (2006) presenta: "buscar, obtener, tratar y usar información, de forma crítica y sistemática, evaluando su pertinencia y diferenciando entre información real y virtual, pero reconociendo sus vínculos. Las personas deben ser capaces de utilizar herramientas para producir, presentar y comprender información compleja, tener la habilidad de acceder a servicios basados en internet y saber utilizar las TIC en apoyo del pensamiento crítico, la creatividad y la innovación. Las TIC requieren una actitud crítica y reflexiva acerca de la información disponible y un uso responsable de los medios interactivos" (Figel, 2006).

- Redes sociales. La red social es un término que procede del latín rete, y hace referencia a la estructura que tiene un patrón característico. El concepto se aplica en diversos ámbitos, como la informática, las comunicaciones, las relaciones interpersonales, entre otros aspectos de la vida del ser humano actual. Las redes sociales en internet fueron creadas con el fin de simplificar y facilitar algunas tareas, como el envío de una mensaje, encontrar trabajo, conocer amigos, encontrar pareja, un lugar para vivir, crear comunidades, creación de contacto directo, cooperación, organización de eventos, entre otras.

De acuerdo a lo expuesto en la Revista Electrónica de Tecnología Educativa EDUTEC por Cascales (2011), las redes sociales son estructuras sociales compuestas de grupos de personas, las cuales están conectadas entre sí, por uno o varios tipos de relaciones e intereses comunes. Igualmente, se encuentra multitud de herramientas y aplicaciones que proporcionan diversas opciones como espacios compartidos, toma de decisiones comunes, asignación de tareas y responsabilidades, votaciones, gestiones de grupo, y muchas otras posibilidades.
Las redes sociales posibilitan mayor comunicación e interacción entre sus miembros, y de acuerdo a Cascales (2011) tienen el propósito de interconectar usuarios que comparten aficiones, amistades, ideas, fotografías, vídeos y elementos multimedia de todo tipo.

Cascales (2011) analiza la filosofía de las redes sociales tomando como base el principio de comunidad abierta y no jerarquizada, que vincula a los usuarios mediante un tema o actividad común y una plataforma web (software social) que posibilita operar de manera sencilla e intuitiva en lo que se conoce como 'ámbito de las 3Cs' (Content, Construction and Colaboration): contenidos libres de derechos de autoría y generados gracias al trabajo individual o colaborativo de los miembros de la red.

De acuerdo a los postulados de Santamaría (2008), las redes sociales son una de las estructuras sociales más potentes e innovadoras para el trabajo en red, que pueden convertirse en comunidades de aprendizaje y en redes de conocimiento y emprendimiento.

Siemens \& Downes a través de la teoría del conectivismo (considerada la teoría del aprendizaje para la era digital (Gutierréz, 2015), explican como la aprehensión del conocimiento se da por medio de las conexiones dentro de las redes. "El modelo utiliza el concepto de una red con nodos y conexiones para definir el aprendizaje. El conectismo integra los principios explorados por la teoría del caos, las redes neuronales, complejidad y autoorganización"

(Siemens, G., 2010, p. 7); y establece como principios del conectivismo los citados a continuación:

- El aprendizaje y el conocimiento yacen en la diversidad de opiniones.

- El aprendizaje es el proceso de conectar nodos o fuentes de información.

- No sólo de los humanos se aprende, el conocimiento puede residir fuera del ser humano.

- La capacidad de aumentar el conocimiento es más importante que lo que ya se sabe.

- Es necesario nutrir y mantener las conexiones para facilitar el aprendizaje continuo.

- La habilidad para ver las conexiones entre los campos, ideas y conceptos es primordial.

- La información actualizada y precisa es la intención de todas las actividades del proceso conectivista. 
La toma de decisiones es en sí misma un proceso de aprendizaje. Escoger qué aprender y el significado de la información entrante es visto a través de la lente de una realidad cambiante. Es posible que una respuesta actual a un problema esté errada el día de mañana bajo la nueva información que se recibe (Siemens, G., 2010, p. 7).

Estos principios, agrupan en sí mismos, las relaciones existentes entre los subsistemas planteados, permitiendo establecer conexiones asertivas, entre los parámetros exigidos por la educación en la media técnica y la utilización de las redes sociales, como medio para generar una cultura emprendedora, que forje la interacción adecuada del individuo dentro de su contexto familiar, social y ambiental en la nueva era. Conceptos clave para el desarrollo de los procesos de la presente investigación.

\section{Método}

La investigación se desarrolla principalmente en tres etapas. La primera, la identificación y descripción de la problemática. Para ello, el trabajo se realiza con los estudiantes de grado décimo y undécimo del Colegio Fernando Mazuera Villegas en la modalidad de Asistencia Administrativa (170 estudiantes), modalidad articulada en formación Técnica, con el Servicio Nacional de Aprendizaje SENA. Su contexto educativo exige el desarrollo de competencias relacionadas con servicio al cliente, producción documental, organización documental, análisis de la información, eventos y emprendimiento. Cada estudiante cuenta con un equipo de cómputo y acceso a internet ilimitado.

En esta primera etapa el instrumento de recolección de información utilizado es la observación directa participativa (como docente). En el trabajo de campo realizado se llevó el debido registro de las ocasiones en que cada joven ingresaba a las redes sociales durante la jornada académica (10 horas semanales), el uso que les daban y cuánto tiempo permanecían en ella. Para llevar éste control, se instaló el Programa Faronic Insicht Teacher ${ }^{10}$, en el compu-

\footnotetext{
${ }^{10}$ Es una eficiente herramienta de gestión de aulas que ayuda a los profesores a crear una mejor experiencia de aprendizaje, devuelve a los profesores el control de las aulas. Utilizando la sencilla consola del profesor puede eliminar distracciones como la navegación web o los juegos en línea. A veces es difícil saber si los estudiantes están realmente trabajando en la lección. Ahora puede confiar en que los estudiantes estarán con sus tareas gracias a las prácticas funciones de supervisión como la capacidad de alternar aleatoriamente las pantallas de los estudiantes para proyectarlas delante de la clase. El chat integrado permite a los profesores y estudiantes tener conversaciones uno a uno sin interrumpir al resto de la clase. Hacer que los estudiantes que se hayan perdi-
}

tador del docente, el cual permitió inicialmente, registrar el proceder de los estudiantes alrededor de los aspectos ya mencionados, sin tomar medidas que coaccionaran su comportamiento habitual.

La mayoría de los estudiantes antes de entrar a su correo electrónico, herramienta utilizada para socializar la agenda diaria, ingresan a su cuenta en Facebook la cual permanece abierta durante toda la jornada, y su uso se limita a chatear con los compañeros del mismo grado, hacer comentarios sobre estados emocionales y compartir fotos de su vida familiar y social. Canalizan su atención en esta herramienta y descuidan las actividades planeadas como parte de su formación técnica, razón por la que en muchas ocasiones el tiempo no le es suficiente para entregar los productos, o en su efecto, los entregan con muy baja calidad, convirtiéndose en una de las razones más influyentes por las que obtienen resultados negativos en los procesos de evaluación.

La minoría restante, utilizan Instagram y Tumblr con los mismos fines y en la misma intensidad que el resto del grupo.

Además de lo anterior, los informes académicos facilitados por la Coordinación de la Media Técnica, demuestran debilidades en el desarrollo de competencias de emprendimiento, instrumentos que permitieron mapear la situación, dando lugar a la etapa descriptiva.

Para la etapa de interpretación, se aplicó una encuesta al 100\% de los estudiantes de la Media Técnica del Colegio Fernando Mazuera Villegas en la modalidad de Asistencia Administrativa. Instrumento elaborado mediante la Técnica del Ciclo Cibernético de transformación (CCT) ${ }^{11}$ y el Revelador del Cociente Tricerebral (CT) ${ }^{12}$ Nivel 1. Herramienta que permitió caracterizar el grupo de estudiantes, interpretando sus facultades capacidades y factores de inteligencia.

El análisis de los datos, realizado desde la teoría tricerebral que propone la cibernética social, evidencia la necesidad de potenciar en el estudiante habilidades que se relacionen con el "hacer", con un vínculo directamente proporcional a la necesidad de que los conocimientos adquiridos se lleven a la práctica e implementen en acciones

do recuperen el hilo es rápido gracias a las funciones de control remoto. Planificar el ritmo perfecto de aprendizaje es posible con las funciones de evaluación y votación que pueden ayudarle a medir el grado de entendimiento del estudiante (Faronics).

\footnotetext{
${ }^{11}$ La secuencia mínima, popular de un cerebro tríadico es: sentir, pensar y actuar (Waldemar, 2002).

${ }^{12}$ Es un espejo del tricerebral, de las competencias individuales, corporativas y étnicas del ser humano (Waldemar, 2002).
} 
de la vida diaria (verdaderas herramientas para la vida, en palabras de Miguel Solano Rector de la Institución para el año 2014), sumado a las exigencias del contexto educativo, permitieron a la investigación enmarcarse dentro de las tres categorías, mencionadas a lo largo del presente artículo: educación (por las nuevas formas de construir conocimiento), comunicación (mal uso de las redes sociales) y producción (la exigencia de la media técnica por desarrollar competencias de emprendimiento). Una vez planteada, argumentada y analizada esta triangulación, el siguiente paso es realizar una propuesta que engrane sistémicamente éstas tres condiciones.

Para la puesta en marcha de su elaboración, se tienen en cuenta tres pilares fundamentales: la estrategia pedagógica debe contemplar las nuevas tendencias para construir conocimiento, debe desmantelar el eficiente uso de las redes sociales, y promover la cultura emprendedora.

En la tercera etapa: la propositiva, se diseña una herramienta pedagógica que reúne información sobre el uso actual que las empresas dan a las redes sociales. Herramienta que describe paso a paso el cómo utilizarlas para promover ideas de negocios, su alcance y beneficio. Diseñada por las dimensiones y niveles de actuación de la teoría tricerebral (Waldemar, 2002) bajo los parámetros dados por la tutora Gladys Cruz de Cidiel (2009), en el módulo "concepto y tendencias de currículo"13, material que fue adaptado y aplicado al manual finalmente titulado: "Manual para el manejo y aplicación pedagógica de las redes sociales en el emprendimiento" (ver Figura 1)

\section{Resultados}

Una vez implementada ésta propuesta, pedagógicamente estructurada, los estudiantes como paso introductorio, mostraron interés colosal por la exploración de estas nuevas herramientas que cotidianamente manejaban en las redes sociales pero sin conocer el impacto de las mismas en el mejoramiento de la calidad de vida.

Para evidenciar la anterior afirmación, y cómo la estrategia se diseñó teniendo en cuenta los parámetros dados por la teoría tricerebral de Waldemar de Gregori, estructura (ver Figura 2) que permite, al terminar cada ciclo, una retroalimentación del mismo, se lleva a cabo la implementación de encuestas en línea que permiten realizar sondeos constantes del nivel de aprehensión del conocimiento que alcanza el estudiante (ver Tabla 2).

\footnotetext{
${ }^{13}$ Módulo cuya temática fue desarrollada cursando la Especialización en Gerencia de Proyectos Educativos en la Universidad Cooperativa de Colombia en el 2009.
}

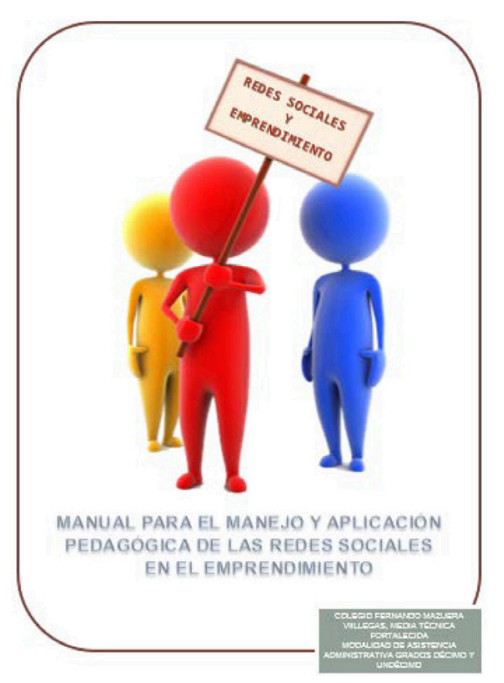

Figura 1. Portada del manual para el manejo y aplicación pedagógica de las redes sociales en el emprendimiento.

Fuente: Gladys Cruz de Cidiel (2009). Módulo. Concepto y tendencias de currículo.

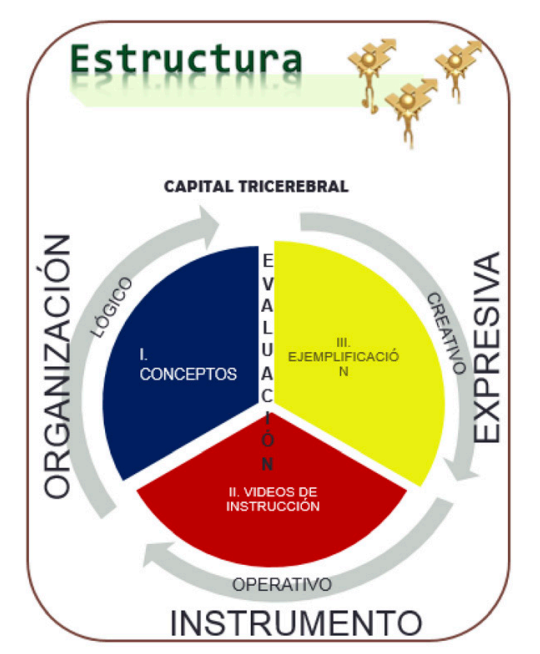

Figura 2. Estructura. Capital tricerebral.

Fuente: Teoría tricerebral de Waldemar de Gregori (2002).

Tabla 2.

Resultados sondeos encuenta on line.

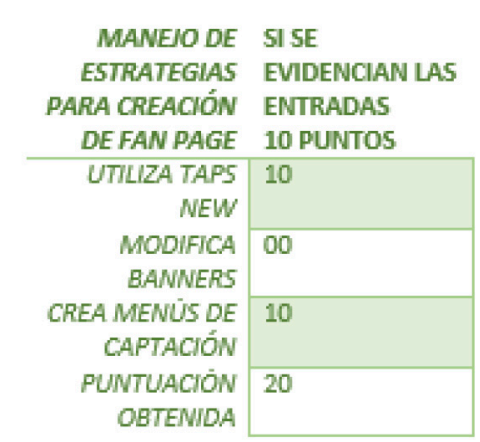

Fuente: elaboración de los autores.

Nota. Aspecto Fan Page cerebro: lógico. 
Por otro lado, la observación directa, después de que los estudiantes desarrollan las actividades propuestas, permite registrar acciones que evidencian el interés por desarrollar técnicas para el manejo de las nuevas herramientas mediante la construcción colectiva de conocimiento, desplazando notoriamente actividades de ocio y generando cultura emprendedora.

Además de lo anterior, fue de gran impacto para ellos descubrir como ambientes virtuales que manipulan diariamente, esconden grandes beneficios para su propio proyecto de vida, relacionando exitosamente los conocimientos que adquieren en el aula de clase con las necesidades propias del "mundo real" (haciendo referencia con éste término al mundo laboral)

\section{Conclusiones}

Partiendo de la pregunta que motiva la investigación: ¿Cómo utilizar en las aulas de clase, las redes sociales para desarrollar competencias de emprendimiento que contribuyan a la gestión del proyecto de vida? Se evidencia que el sistematizar, en un manual, información alrededor de las herramientas que proporcionan las redes sociales para generar ingresos, posicionar ideas de negocio y comercializar bienes y servicios, despiertan en el joven estudiante, entusiasmo por ahondar en este campo del conocimiento. Situación que genera beneficios colaterales, debido a que para tal fin, debe desarrollar habilidades comunicativas, trabajar en equipo, invertir el tiempo de conexión en la exploración de nuevas técnicas, retroalimentar sus aciertos y desaciertos, liderar y hacer seguimiento de procesos, innovar, poner a prueba las capacidades que lo caracterizan por pertenecer a la generación de los llamados "nativos digitales", romper con cadenas locales y abrir su visión a dimensiones panorámicas que le permitan incursionar en un mundo ya globalizado.

Es de aclarar, que los resultados de la investigación generaron tal impacto, además, por lo novedoso de la información y la herramienta utilizada para socializarla.

En el proceso enseñanza-aprendizaje es relevante la aplicación de la teoría, en actividades ligadas a la realidad, aspecto que se sugiere tener en cuenta para nuevas investigaciones. Fue muy novedoso para los estudiantes evidenciar que efectivamente un youtubers gana dinero en línea, que creando una fan page, obtiene clientes para comercializar bienes y servicios, que una cuenta Pay Pal, le permite manejar dinero en línea, que el botón PSE le permite a sus clientes agregar productos al carrito, que a través de Google Adsense puedes generar ingresos, y que su negocio puede ser ubicado por el mundo entero, entre otras de las muchas ventajas que ofrecen las herramientas virtuales. De ahí, la sugerencia para investigaciones que giren alrededor de desarrollar nuevas estrategias pedagógicas, de engranar cuidadosamente la teoría con la razón de ser en la vida real del estudiante. Esto es, propender por brindar verdaderas herramientas para la vida y no el simple desagüe de teorías plasmadas en una malla curricular.

\section{Referencias}

ABC de las Redes Sociales en internet. (2 de abril, 2015). Los tiempos.com. Obtenido de http://m.lostiempos.com/oh/actualidad/actualidad/20110102/abc-de-las-redes-sociales-eninternet_106593_208639.html

Ahumada Méndez, L. S. (2013). Las competencias aplicadas al emprendimiento.

Aldrich, H. (2012). The emergence of entrepreneurship as an academic field: A personal essay on. Elsevier B.V, 1240 - 1248.

Arias, C. y. (2011). La educación para el emprendimiento y empresarismo virtual. Barranquilla: Universidad Católica del Norte.

Barrueta, H. (12 de julio, 2010). eluniversal.com. Recuperado el 15 de 01 de 2015, de http://www.eluniversal.com.mx/articulos/59558.html

Baiget, T. (2009). Networking y comunidades en la web social. Tres proyectos para mejorar la comunicación.

Bilbao, A. (2002). Rasgos y actitudes de los emprendedores. Caracas: Corporación Andina de Fomento - Venezuela Competitiva.

Buckingham, D. (2002). Crecer en la era de los medios electrónicos. Tras la muerte de la infancia. Madrid: Morata.

Cameron, K. (1999). Diagnosing and changing organizational culture: Based on the competing values. Framework, AddisonWesley Reading M.A.

Cascales, A. (2011). www.edutec.com. Recuperado el Febrero de 2015, de Revista electrónica de Tecnología No.58: http://edutec.rediris.es/Revelec2/Revelec38/pdf/Edutec-e_38_Cascales_Real_Marcos.pdf

Cayambe, C. (2012). Universidad Técnica del Norte, Perú. Recuperado el 2 de Abril de 2015, de repositorio.utn.edu.ec/bitstream/.../1655/1/FECYT\%201389\%20TESIS.pdf

Castilla, E. (10 de junio, 2011). Ebanking News. Recuperado el Junio de 2015, de http://www.ebankingnews.com/noticias/ marketing-4-0-realidad-o-ficcion-006535

Chomsky, N. (1957). Estructuras Sintácticas. México: Siglo Veintiuno Editores.

Colegio Fernando Mazuera Villegas. (2014). Informe Media Fortalecida. Bogotá D.C. 
CIENCIA Y PODER AÉREO | Revista Científica de la Escuela de Postgrados de la Fuerza Aérea Colombiana | Vol. 11 | Enero - Diciembre de 2016

Colombia, P. N. (02 de enero, 2015). Ministerio de Defensa Policía Nacional de Colombia. Obtenido de (http://www.policia. gov.co/portal/page/portal/UNIDADES_POLICIALES/Direcciones_tipo_Operativas/Direccion_Seguridad_Ciudadana/ Planes_de_Seguridad/Recomendaciones_de_seguridad/ delitos_informaticos)

Colombia, B. M. (febrero, 2008). Cippec.org. Obtenido de http:// cippec.org/mapeal/wp-content/uploads/2014/05/BANCOMUNDIAL-COLOMBIA-La-calidad-de-la-educ-en-COI.pdf

Comunicaciones, M. D. (05 de mayo, 2014). mintic.gov.co. Recuperado el 13 de 01 de 2015, de http://www.mintic.gov.co/ portal/604/w3-article-6048.html

Clements, P. (2002). Líneas de productos software: prácticas y patrones. Boston: Adisson-Weslwy.

Ciclotics. (2011). Obtenido de http://wwwciclotics.blogspot.com/

Cidiel, G. C. (2009). Concepto y tendencias de currículo. Universidad Cooperativa de Colombia.

De Haro, J. (2010). Manual imprescindible redes sociales para la educación. Madrid, España: Ediciones anaya.

Distrital, S. D. (2015). Sedbogotá. Obtenido de http://www.sedbogota.edu.co/index.php/educacion-superior/116-educacionmedia-y-superior/2127-proyecto-891.html

Drucker, P. (1969). La era de la discontinuidad. En La era de la discontinuidad. Barcelona: Información Comercial Española.

Enciso, B. (2012). Plan de Negocios y Soluciones NTIC. Bogotá D.C.: Universidad EAN Bogotá.

Espectador, E. (2014). El Espectador. Obtenido de www.elespectador.com.co

Facebook.com. (2014). Mesa de ayuda. Obtenido de www.facebook.com: http://www.facebook.com/help/?page=18960

Faronics, P. (s.f.). Faronics. Obtenido de http://www.faronics.com/ es/products/insight/

Figel, J. (2006). Competencias clave para el aprendizaje permanente - un marco europeo. Obtenido de http://es.scribd.com/ doc/84947565/Marco-Europeo-Competencias-Clave-ParaEl-Aprendizaje-Permanente

Formichella, M. (1 de abril, 2010). El concepto de emprendimiento y su relación con la educación, el empleo y el desarrollo local. Obtenido de INTA.com: ftp://ftp.unicauca.edu.co/Facultades/FIET/Materias/Gestion_tecnologica/2005/Clase\%2012/ Nuevos/emprendydesarrollolocal.pdf

García, L. (2006). Técnicas y habilidades directivas.

Graells, M. (2005). Cambios en los centros educativos: construyendo la escuela del futuro. In Didáctica, Innovación y Multimedia.

Gutierréz, F. G. (21 de agosto, 2015). Infotecarios. Obtenido de http://www.infotecarios.com/bibliotecas-y-aprendizaje-digital-el-conectivismo/
Healy, J. (1998). No se puede conectar. Nueva York: Simon and Shuster.

Herrera, H. (2009). Investigación sobre Redes Sociales y Emprendimiento. Revisión de la literatura y agenda futura. Innovar de Ciencias Administrativas. Universidad Nacional de Colombia .

Instagram.com. (2015). https://instagram.com/. Obtenido de https://instagram.com/about/us/

IESE. (2008). Emprendimiento: conceptos básico en competencias. Instituo de Estudios Técnicos en Educación.

Irurzun, J. y. (2012). Empresarios y redes empresariales en la andalucía contemporánea. Universidad de Granada. Obtenido de https:// www.academia.edu/5713797/Empresarios_y_redes_empresariales_en_la_Andaluc\%C3\%ADa_contempor\%C3\%A1nea

Josean, I. (2012). Empresarios y redes empresariales en la Andalucía contemporánea.

Jóvenes en el siglo XXi, M. Jóvenes en el siglo XXi, M. (2009). En Usos de las TIC, Relaciones sociales y cambios en la socialización de las y los jóvenes. Juventud y nuevos medios de comunicación.

Lambing, P. (1988). Empresarios medianos y pequeños. Mexico: Prentice Hall.

Linkedin. (2015). Linkedin.com. Recuperado el 3 de Abril de 2015, de https://ayuda.linkedin.com/app/answers/detail/a_ id/34919

Lederman, D. (2014). El emprendimiento en América Latina. Muchas empresas y poca innovación.

LLera, B. (2003). Impacto de las TIC en la educación: funciones y limitaciones Graells.P.M. (2013). Artículo 3c TIC: cuadernos de desarrollo aplicados a las TIC, 2(1), 2.

Ley 1014 de 2006. Ley de Fomento de la cultura del emprendimiento. (2006). Bogotá: República de Colombia.

Martínez, M. (2014). Redes sociales y TIC, su papel en la educación del siglo XX1.

Ministerio de Tecnologías de la Información y las Comunicaciones. (05 de 05 de 2014). mintics.gov.co. Recuperado el 13 de 01 de 2015, de http://www.mintic.gov.co/portal/604/w3article-6048.html

Ministerio de Educación y Ciencia. (s.f.). books.google.es. Recuperado el 15 de 01 de 2015, de https://books.google.es/

Mineducación. (2006). Articulación de la educación con el mundo productivo. Obtenido de http://www.mineducacion.gov. co/1621/articles-106706_archivo_pdf.pdf

Muñoz, C. (2012). El líder y sus características de buen motivador. Obtenido de http://carlosmunozv.blogspot.com/2012/12/ el-lider-y-sus-caracteristicas-de-buen.html

Naciones Unidas. (2004). Naciones Unidas. Juventud y tecnologías de la información. Obtenido de http://www.euskadi.net/r33- 
2732/es/contenidos/informacion/observ_juv_otros_documentos/es_5986/adjuntos/juventud_y_tecnologias_de_la_ informacion_c.pdf

Núñez, P. T. (2012). La profesión en el aula. Cómo investigar y mejorar. (U. C. Madrid, Ed.) La profesión en el aula. Cómo investigar y mejorar. Universidad Complutense de Madrid , 18, 951 - 957.

Páez, L. (2004). Liderazgo: evolución y conceptualización. Bogotá: Universidad Externado de Colombia.Papert, S. (1995). La máquina de los niños. Replantearse la Educación en la era de los ordenadores. Barcelona: Paidós.

Portafolio. (02 de 12 de 2012). El portafolio.co. Obtenido de http:// www.portafolio.co/portafolio-plus/internet-la-oportunidadun-negocio-crecimiento

Prensky, M. (2011). Enseñar a nativos digitales. Ediciones SM.

Redes sociales blogspot.com. (2 de abril, 2015). Obtenido de http:// redes0cial.blogspot.com/p/ejemplos-de-aplicacion.html

Rodríguez, A. (2007). Articulación de la Educación Mediacon la Educación Superior y el mundo del trabajo. Lineamientos Generales, 55. Bogotá D.C., Colombia: Servi Offset.

Rodríguez, N. (2012). Sistematización de iniciativas y propuestas de emprendedurismo. Lima: Consejo Nacional de Competitividad del Ministerio de Educación del Perú.

Rueda, J. (2007). Revista de ciencias sociales. Obtenido de Apostadigital.com: http://www.apostadigital.com/revistav3/hemeroteca/jjrueda.pdf

Santamaría, F. (2008). Posibilidades Pedagógicas, redes sociales y Comunidades educativas. Cuadernos de Comunicación. Telos.

SENA. (2014). Cartilla mentalidad emprendedora. Bogotá: Servicio Nacional de Aprendizaje.

Serrano, J. E. (2012-2013). www.uji.es. Obtenido de http://www3. uji.es/ betoret/Instruccion/Aprendizaje\%20y\%20DPersonalidad/Curso\%2012-13/Apuntes\%20Tema\%201\%20La\%20 transicion\%20adolescente\%20y\%20la\%20educacion.pdf

Siemens, G. (2010). Capítulo 5 Conectivismo: una teoría de aprendizaje para la era digital. En G. Siemens. Conectados en el Ciberespacio. Madrid: UNED.

Soto, A. (2000). Educación en tecnología. Un reto y una exigencia social. Bogotá D.C., Colombia: Editorial Magisterio.

Trias de Bes, F. (2007). El libro negro del emprendedor. No digas que nunca te lo advirtieron. Madrid: Empresa Activa.

Tumblr. (2015). tumblr.com. Recuperado el 3 de Abril de 2015, de https://www.tumblr.com/

Velandia, C. (2006). 2. Subsistemas. En C. Velandia. Metodología Interdisciplinaria, 24-26. Bogotá.

Waldemar, G. E. (2002). Capital tricerebal. Bogotá: Beta.
Yahoo.com. (2 de abril, 2015). www.yahoo.com. Obtenido de yahoo respuestas: https://espanol.answers.yahoo.com/question/index?qid=20110523205240AAO6SMG 